\title{
Subcommuting and comparable iterative roots of order preserving homeomorphisms
}

Subcommuting \section{and \\ comparable iterative roots}

\author{
Veerapazham Murugan \\ Department of Mathematical and Computational Sciences, \\ National Institute of Technology Karnataka, Surathkal, Mangalore, India, and \\ Murugan Suresh Kumar \\ Department of Mathematics, The Gandhigram Rural Institute, Gandhigram, India
}

Abstract

\begin{abstract}
It is known that the iterative roots of continuous functions are not necessarily unique, if it exist. In this note, by introducing the set of points of coincidence, we study the iterative roots of order preserving homeomorphisms. In particular, we prove a characterization of identical iterative roots of an order preserving homeomorphism using the points of coincidence of functions.
\end{abstract}

Keywords Iterative roots, Homeomorphisms, Commuting functions, Subcommuting functions,

Comparable functions

Paper type Orginal Article

\section{Introduction}

Given a function $F: X \rightarrow X$ and a positive integer $n$, if there is a function $f: X \rightarrow X$ such that

$$
f^{n}(x)=F(x), \quad \text { for all } x \in X
$$

(where $f^{n}$ is $n$ times composition of $f$ ) then $f$ is called an $n$th iterative root or fractional iterate of order $n$ of $F$. The problem of finding the iterative root of functions was initiated in the classical works of Charles Babbage [1]. The iterative roots of continuous monotone and piecewise monotone function was developed in the works of Bödewadt [2], Lojasiewicz [7], Kuczma [4], Zhang [6,12] and many others. For a detailed study of recent results on iterative roots of continuous piecewise monotone functions can be found in the survey paper by Zdun and Solarz [11].

\footnotetext{
JEL Classification - 39B12, 39B22

(C) Veerapazham Murugan and Murugan Suresh Kumar. Published in Arab Journal of Mathematical Sciences. Published by Emerald Publishing Limited. This article is published under the Creative Commons Attribution (CC BY 4.0) license. Anyone may reproduce, distribute, translate and create derivative works of this article (for both commercial and non-commercial purposes), subject to full attribution to the original publication and authors. The full terms of this license may be seen at http:// creativecommons.org/licences/by/4.0/legalcode

Declaration of Competing Interest: The authors declare that they have no known competing financial interests or personal relationships that could have appeared to influence the work reported in this paper.

The publisher wishes to inform readers that the article "Subcommuting and comparable iterative roots of order preserving homeomorphisms" was originally published by the previous publisher of the Arab Journal of Mathematical Sciences and the pagination of this article has been subsequently changed. There has been no change to the content of the article. This change was necessary for the journal to transition from the previous publisher to the new one. The publisher sincerely apologises for any inconvenience caused. To access and cite this article, please use Murugan, V., Kumar, M.S. (2019), "Subcommuting and comparable iterative roots of order preserving homeomorphisms", Arab Journal of Mathematical Sciences, Vol. 26 No. 1/2, pp. 203-210. The original publication date for this paper was 31/10/2019.
}

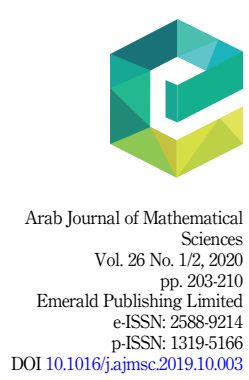


AJMS

$26,1 / 2$

204

For the class of strictly increasing continuous functions, we have the following result.

Theorem 1.1 ([5]). Let $I \subseteq \mathbb{R}$ be any interval. Then every strictly increasing continuous function $F$ from I into itself possesses a strictly increasing continuous iterative roots of order $n$, for all $n \in \mathbb{N}$.

Theorem 1.1 guarantees the existence of strictly increasing continuous iterative roots of a strictly increasing continuous functions. Moreover, this strictly increasing continuous $n$th order iterative root depends on arbitrary strictly increasing homeomorphisms (see Theorem 11.2.2 [5]), and hence its iterative roots are not necessarily unique. In fact, every strictly increasing continuous function, other than identity, possesses infinitely many strictly increasing continuous $n$th order iterative roots.

In fact, uniqueness of iterative roots of a special class of monotonic functions was conjectured by Bödewadt [2] and answered in negative by Smajdor [9]. Motivated by Bödewadt, suppose $f$ and $g$ are two iterative roots of order $n$ of a strictly increasing homeomorphism $F$ (i.e. $f^{n}=g^{n}=F$ ), it is reasonable to ask under what condition $f$ and $g$ are identically equal?

It is known that, if $f^{n}=g^{n}=F$ and $f, g$ commutes each other (i.e. $f g=g f$ ) then $f$ must be equal to $g$ (see [10]). In this article, we further investigate this problem. We give some sufficient conditions, using the set of points of coincidence of two functions. Also, for given order preserving homeomorphism from an interval onto itself, by generalizing the result in [10], we characterize the conditions of identical iterative roots of an order preserving homeomorphism.

\section{Set of points of coincidence}

Throughout our discussion we fix $I=(a, b)$, where $-\infty \leq a \leq b \leq \infty$, and $H(I)$ denotes the set of all order preserving homeomorphisms from $I$ onto itself. Here after we always assume all the functions are in the class $H(I)$ unless otherwise stated.

Let $f$ and $g$ be two order preserving homeomorphisms from the interval $I$ onto $J \subseteq I$. We say $f$ and $g$ are comparable, if either $f(x) \leq g(x)$ or $g(x) \leq f(x)$ for all $x \in I$, and if the inequalities are strict then we say $f$ and $g$ are strictly comparable.

Proposition 2.1. If $f$ and $g$ are two strictly comparable order preserving homeomorphisms from I onto $J \subseteq I$, then $f^{n}$ and $g^{n}$ are strictly comparable order preserving homeomorphisms, for all $n \in \mathbb{N}$. In addition to that, if $J=I$ then $f^{-n}$ and $g^{-n}$ are also strictly comparable order preserving homeomorphisms, for all $n \in \mathbb{N}$.

Proof. First we prove the result for positive integers using induction on $n$. Assume $f(x)<g(x)$ for all $x \in I$. Suppose there exists $t \in f(I)$ such that $f^{2}(t) \geq g^{2}(t)$. Since $f(t)<g(t)$ we have $f(f(t))<f(g(t))$. Therefore

$$
\begin{gathered}
g(g(t)) \leq f(f(t))<f(g(t)) . \\
\text { i.e., }(f-g)(g(t))>0 .
\end{gathered}
$$

Since $(f-g)(t)<0$, by intermediate value theorem there exists $c \in(t, g(t))$ such that $f(c)=g(c)$, which is a contradiction. Hence $f^{2}<g^{2}$ on $(a, b)$.

Assume $f^{k}(x)<g^{k}(x)$ for all $x \in(a, b)$ and $1 \leq k \leq n-1$. Suppose there is a $t \in f^{n-1}(I)$ such that

$$
f^{n}(t) \geq g^{n}(t) .
$$

Since $f^{n-1}(t)<g^{n-1}(t)$ we have $f^{n}(t)<f\left(g^{n-1}(t)\right)$. Therefore

$$
\begin{gathered}
g^{n}(t) \leq f^{n}(t)<f\left(g^{n-1}(t)\right) . \\
i . e .,(f-g)\left(g^{n-1}(t)\right)>0 .
\end{gathered}
$$

Since $(f-g)(t)<0$, by intermediate value theorem there exists $c \in\left(t, g^{n-1}(t)\right)$ such that $f(c)=g(c)$, which is a contradiction. Hence $f^{n}<g^{n}$ on $(a, b)$. 
Now, we prove the result for negative integers by assuming $J=I$. First we prove if $f(x)<g(x)$ for all $x \in I$, then $g^{-1}(x)<f^{-1}(x)$ for all $x \in I$. Suppose there is a $t \in I$ such that $g^{-1}(t) \geq f^{-1}(t)$. If $g^{-1}(t)=f^{-1}(t)$ then there exists $x \in I$ such that $f(x)=g(x)=t$, which is not possible as $f(x)<g(x)$ for all $x \in I$. Therefore $g^{-1}(t)>f^{-1}(t)$. But this implies

$$
f\left(g^{-1}(t)\right)>t>g\left(f^{-1}(t)\right) .
$$

Since $f\left(g^{-1}(t)\right)<g\left(g^{-1}(t)\right)$ we have $t>f\left(g^{-1}(t)\right)>t$, which is a contradiction. Thus $g^{-1}(x)<f^{-1}(x)$ for all $x \in I$. Therefore, as above $g^{-n}(x)<f^{-n}(x)$ for all $x \in I$ and for all $n \in \mathbb{N}$.

For any two functions $f$ and $g$, we denote the set of points of coincidence of $f$ and $g$ by $Z(f, g)$. i.e., $Z(f, g)=\{x \in I \mid f(x)=g(x)\}$.

Theorem 2.2. If $Z(f, g)$ is a finite set, then $f^{n} \neq g^{n}$ for all $n \in \mathbb{Z} \backslash\{0\}$.

Proof. If $Z(f, g)$ is empty, then either $f(x)<g(x)$ or $g(x)<f(x)$ for all $x \in I$. Therefore by Proposition 2.1, $g^{n}(x) \neq f^{n}(x)$ for all $x \in I$ and for all $n \in \mathbb{Z} \backslash\{0\}$.

On the other hand, if $Z(f, g)$ is non empty, we argue as follows:

If $f$ and $g$ do not have a common fixed point, then there exists $t \in I$ such that $f(t)=t$ but $g(t) \neq t$. Without loss of generality, let $g(t)<t$. Therefore $g^{n}(t)<t$ but $f^{n}(t)=t$ which in turn implies $f^{n} \neq g^{n}$ for all $n \in \mathbb{Z}$.

If $f$ and $g$ have common fixed points, then the set $\{x \in I \mid f(x)=g(x)=x\}$ must be finite. Let $\alpha_{i}$ where $1 \leq i \leq k$ be the common fixed points of $f$ and $g$ with

$$
a=\alpha_{0}<\alpha_{1}<\cdots<\alpha_{k}<\alpha_{k+1}=b .
$$

Now, to prove our result it is enough to prove $f^{n} \neq g^{n}$ on $\left(\alpha_{i}, \alpha_{i+1}\right)$ for some $i$. Since on each $\left(\alpha_{i}, \alpha_{i+1}\right)$ both the functions $f$ and $g$ are self maps and has no fixed points, we may assume $f$ and $g$ do not have fixed points in $I$.

Case 1. $x<f(x)$ and $g(x)<x$ for all $x \in I$.

Since $g(x)<x<f(x)$ for all $x \in(a, b)$, for any positive integer $n, g^{n}(x)<x<f^{n}(x)$. Moreover for any positive integer $n, f^{-n}(x)<x<g^{-n}(x)$ for all $x \in(a, b)$ as $f^{-1}(x)<x<g^{-1}(x)$ for all $x \in(a, b)$. Hence for any $n \in \mathbb{Z} \backslash\{0\}, f^{n} \neq g^{n}$.

Case 2. $x<f(x)$ and $x<g(x)$ for all $x \in I$.

Step 1: We prove the result for positive integers.

Let $\alpha=\max \{x \in(a, b) \mid f(x)=g(x)\}$, then $f(\alpha)=g(\alpha)$ and $f(x) \neq g(x)$ for all $x \in(\alpha, b)$. Without loss of generality assume $f(x)<g(x)$ for all $x \in(\alpha, b)$. To prove $f^{n} \neq g^{n}$ on $I$, we prove $f^{n}<g^{n}$ on $(\alpha, b)$ for all $n \in \mathbb{N}$.

Since $f$ and $g$ are self maps on $(\alpha, b)$, By Proposition 2.1, $f^{n}(x)<g^{n}(x)$ for all $x \in(\alpha, b)$.

Step 2: We prove the result for negative integers.

Let $\beta=\min \{x \in(a, b) \mid f(x)=g(x)\}$, then $f(\beta)=g(\beta)$ and $f(x) \neq g(x)$ for all $x \in(a, \beta)$. We may assume $f(x)<g(x)$ for all $x \in(a, \beta)$. Since

$$
x<f(x)<g(x) \text { for all } x \in(a, \beta),
$$

replacing $x$ by $g^{-1}(x)$ in Eq. (2) we get $g^{-1}(x)<f\left(g^{-1}(x)\right)<x$ for all $x \in\left(a, g^{-1}(\beta)\right)$. In particular,

$$
g^{-1}(x)<f^{-1}(x) \quad \text { for all } x \in\left(a, g^{-1}(\beta)\right) .
$$

To prove $f^{-n} \neq g^{-n}$ on $I$, we prove $g^{-n}<f^{-n}$ on $\left(a, g^{-1}(\beta)\right)$ for all $n \in \mathbb{N}$. Since, both $f^{-1}$ and $g^{-1}$ are self maps on $\left(a, g^{-1}(\beta)\right)$, by Proposition $2.1, g^{-n}(x)<f^{-n}(x)$ for all $x \in\left(a, g^{-1}(\beta)\right)$.

Moreover the cases $f(x)<x$ and $x<g(x)$ for all $x \in I$ and $f(x)<x$ and $g(x)<x$ for all $x \in I$ are similar to case 1 and case 2 .

Lemma 2.3. If $f g=g f$, then $f^{n} g^{m}=g^{m} f^{n}$ for all $n, m \in \mathbb{Z}$. 
Proof. First we prove $f^{n} g=g f^{n}$ for all $n \in \mathbb{Z}$. As $f$ and $g$ commute, we see that

$$
f^{2} g=f(f g)=f(g f)=(g f) f=g f^{2} .
$$

Assume $f^{k} g=g f^{k}$ for all $1 \leq k \leq n-1$. Again, by using induction hypothesis and $f$ and $g$ commute, we see that

$$
g f^{n}=\left(g f^{n-1}\right) f=\left(f^{n-1} g\right) f=f^{n-1}(g f)=f^{n-1}(f g)=f^{n} g .
$$

Therefore $f^{n} g=g f^{n}$ for all $n \in \mathbb{N}$. Since $g f=f g$, pre-multiplying by $f^{-1}$ we get $f^{-1} g f=g$. Now, post multiply by $f^{-1}$ to get $f^{-1} g=g f^{-1}$. Hence by repeating the above process we get $f^{-n} g=g f^{-n}$. Therefore $f^{-n} g=g f^{-n}$ for all $n \in \mathbb{Z}$.

Since $f^{n} g=g f^{n}$ for each $n \in \mathbb{Z}$, again by above argument, we have $f^{n} g^{m}=g^{m} f^{n}$ for all $m \in \mathbb{Z}$.

Proposition 2.4. If $x \in Z(f, g)$ and $f g=g f$, then $f^{n}(x), g^{n}(x) \in Z(f, g)$ for all $n \in \mathbb{Z}$.

Proof. For $x \in Z(f, g)$, we have $f(f(x))=f(g(x))=g(f(x))$. Therefore $f(x) \in Z(f, g)$. By repeating the above process we see that $f^{n}(x) \in Z(f, g)$ for all $n \in \mathbb{N}$. Now, by applying Lemma 2.3, we see that

$$
f\left(f^{-1}(x)\right)=f^{-1}(f(x))=f^{-1}(g(x))=g\left(f^{-1}(x)\right) .
$$

Therefore $f^{-1}(x) \in Z(f, g)$. Hence, by above argument, $f^{-n}(x) \in Z(f, g)$ for all $n \in \mathbb{N}$. i.e., $f^{n}(x) \in Z(f, g)$ for all $n \in \mathbb{Z}$. Similarly $g^{n}(x) \in Z(f, g)$ for all $n \in \mathbb{Z}$.

Theorem 2.5. If $g=g f$, then $Z(f, g)=Z\left(f^{n}, g^{n}\right)$ for all $n \in \mathbb{Z} \backslash\{0\}$

Proof. Step 1: We prove $Z(f, g)=Z\left(f^{n}, g^{n}\right)$ for all $n \in \mathbb{N}$ using induction on $n$. First we prove $Z(f, g)=Z\left(f^{2}, g^{2}\right)$.

For $x \in Z(f, g)$, we have

$$
f^{2}(x)=f(f(x))=f(g(x))=g(f(x))=g(g(x))=g^{2}(x) .
$$

Let $x \in Z\left(f^{2}, g^{2}\right)$. If $f(x) \neq g(x)$, without loss of generality, say $f(x)<g(x)$ then

$$
f^{2}(x)<f(g(x))=g(f(x))=g^{2}(x)
$$

which is not possible. Therefore $Z(f, g)=Z\left(f^{2}, g^{2}\right)$.

Assume $Z(f, g)=Z\left(f^{k}, g^{k}\right)$ for $2 \leq k \leq n-1$. Therefore, by applying Proposition 2.4, for $x \in Z(f, g)$, we have

$$
f^{n}(x)=f^{n-1}(f(x))=f^{n-1}(g(x))=g^{n-1}(g(x)) .
$$

This shows that $Z(f, g) \subseteq Z\left(f^{n}, g^{n}\right)$. Suppose $x \in Z\left(f^{n}, g^{n}\right)$ with $f(x)<g(x)$. Then, by applying Lemma 2.3

$$
f^{n}(x)<f^{n-1}(g(x))=g^{n-1}(f(x))=g\left(g^{n-1}(x)\right)=g^{n}(x),
$$

which is not possible. Therefore $Z\left(f^{n}, g^{n}\right) \subseteq Z(f, g)$. This completes the proof of step 1 .

Step 2: We prove $Z(f, g)=Z\left(f^{-n}, g^{-n}\right)$ for all $n \in \mathbb{N}$.

It is clear from Step 1 that, $Z\left(f^{-1}, g^{-1}\right)=Z\left(f^{-n}, g^{-n}\right)$ for all $n \in \mathbb{N}$. Therefore to prove Step 2, it is enough to prove $Z(f, g)=Z\left(f^{-1}, g^{-1}\right)$.

Let $x \in Z(f, g)$. Suppose $f^{-1}(x)<g^{-1}(x)$. Then, by applying Lemma 2.3 we see that,

$$
x<f\left(g^{-1}(x)\right)=g^{-1}(f(x))=g^{-1}(g(x))=x .
$$

which is not possible. On the other hand, if $g^{-1}(x)<f^{-1}(x)$ then

$$
x<g\left(f^{-1}(x)\right)=f^{-1}(g(x))=f^{-1}(f(x))=x,
$$


again a contradiction. Therefore $f^{-1}(x)=g^{-1}(x)$ whenever $f(x)=g(x)$, i.e. $Z(f, g) \subseteq$ $Z\left(f^{-1}, g^{-1}\right)$. Now by replacing $f$ and $g$ by $f^{-1}$ and $g^{-1}$ respectively, we get $Z(f, g)=Z\left(f^{-1}, g^{-1}\right)$.

Corollary 2.6. If $f, g$ satisfy $f g=g f$ and $f^{n}=g^{n}$ for some $n \in Z$ then $f=g$.

Proof. Since $f g=g f$, by Theorem 2.5, we have $Z\left(f^{n}, g^{n}\right)=Z(f, g)$. But $Z\left(f^{n}, g^{n}\right)=I$ as $f^{n}=g^{n}$. Therefore $f=g$ on $I$.

Theorem 2.7. Let $f, g \in H(I)$ without fixed points such that $f g=g f$. Suppose $Z\left(f^{n}, g^{n}\right)$ is an interval for some $n \in \mathbb{Z}$, then $f=g$ on $I$.

Proof. Since $f g=g f$, by Theorem $2.5, Z(f, g)=Z\left(f^{n}, g^{n}\right)$. Without loss of generality, let $\alpha \in Z(f, g)$ such that $\alpha<f(\alpha)$. Also by Proposition 2.4, $f(\alpha) \in Z(f, g)$. Since $f^{m}(\alpha) \rightarrow b$ and $f^{-m}(\alpha) \rightarrow a$ as $m \rightarrow \infty$. Therefore

$$
I=(a, b)=\bigcup_{m \in \mathbb{Z}}\left[f^{m}(\alpha), f^{m+1}(\alpha)\right] .
$$

Let $y \in\left[f^{m}(\alpha), f^{m+1}(\alpha)\right]$ be arbitrary. Then there is an element $x \in[\alpha, f(\alpha)]$ such that $y=f^{n}(x)$. Since $f=g$ on $[\alpha, f(\alpha)]$ we have $y=f^{m}(x)=g^{m}(x)$. Therefore, by Lemma 2.3,

$$
f(y)=f\left(g^{m}(x)\right)=g^{m}(f(x))=g^{m}(g(x))=g\left(g^{m}(x)\right)=g(y) .
$$

This completes the proof.

\section{Subcommuting and comparable iterative roots}

Definition 3.1 ([3]). Let $f$ and $g$ be two order preserving homeomorphisms on $I$. We say $f$ subcommutes with $g$ if $f(g(x)) \leq g(f(x))$, for all $x \in I$.

Note that every commuting functions are subcommuting, but the converse is not necessarily true. For example, consider the functions $f, g:(0, \infty) \rightarrow(0, \infty)$ by $f(x)=2 x$ and $g(x)=x^{2}$. Clearly $f$ subcommutes with $g$ as $f(g(x))=2 x^{2} \leq g(f(x))=4 x^{2}$ for all $x \in(0, \infty)$. But $f(g(x))=2 x^{2} \neq g(f(x))=4 x^{2}$ for all $x \in(0, \infty)$.

Let $F: I \rightarrow I$ be an order preserving homeomorphism. We prove that it is not possible to have different iterative roots of $F$ which are either comparable or subcommuting.

Theorem 3.2. Let $F \in H(I)$. Suppose $f, g \in H(I)$ satisfy $f^{n}=g^{n}=F$ for some $n \in \mathbb{Z}$. Then the following are equivalent.

1. $f$ subcommutes with $g$.

2. $f$ and $g$ are comparable.

3. $f=g$.

Proof. $\mathbf{3}$ implies $\mathbf{1}$ and $\mathbf{2}$ are trivial.

$(\mathbf{1} \Rightarrow \mathbf{3})$ In view of Corollary 2.6, it is enough to prove that $f g=g f$ on $I$.

Suppose $f g(x)<g f(x)$ for some $x$. Then

$$
\begin{aligned}
g^{n+1}(x) & =g^{n}(g(x)) \\
& =f^{n}(g(x)) \\
& =f^{n-1}(f(g(x))) \\
& <f^{n-1}(g(f(x))) \\
& \leq f^{n-2}\left(g\left(f^{2}(x)\right)\right) \\
& \vdots \\
& \leq g\left(f^{n}(x)\right) \\
& =g^{n+1}(x) .
\end{aligned}
$$

i.e., $g^{n+1}(x)<g^{n+1}(x)$, a contradiction. Hence $f g=g f$. Therefore by Corollary $2.6, f=g$ on $I$. 
AJMS

$26,1 / 2$

208

$(2 \Rightarrow 3)$ Assume $f \leq g$. If possible, let $f(t) \neq g(t)$ for some $t \in I$, therefore $f(t)<g(t)$. Since $f^{n}=g^{n}$, we have

$$
g^{n}(t)=f^{n}(t)<f^{n-1}(g(t)) \leq g\left(f^{n-2}(g(t))\right),
$$

where the last inequality holds since $f \leq g$. But then $g^{n-1}(t)<f^{n-2}(g(t))$ as $g^{-1}$ is an orderpreserving homeomorphisms. Now

$$
g^{n-1}(t)<f^{n-2}(g(t)) \leq g\left(f^{n-3}(g(t))\right),
$$

since $f \leq g$. This implies $g^{n-2}(t)<f^{n-3}(g(t))$, since $g^{-1}$ is an order-preserving homeomorphisms. Continuing this process up to $(n-2)$ times we get

$$
g(g(t))<f(g(t))
$$

a contradiction to our assumption. Therefore $f=g$ on $I$.

Part of a theorem due to McShane [8] is observed below.

Corollary 3.3 ([8]). The only order preserving iterative root of any order of the identity function on $\mathbb{R}$ is the identity function.

Proof. Clearly, identity function is an iterative root of any order of the identity function, it follows from Theorem 3.2, that any order preserving homeomorphism whose iteration is identity becomes identity, as the identity function subcommutes (also commutes, so Corollary 2.6 also applicable) with any function.

Further, if $f \in H(I)$ such that $f^{n}(x)=x$ for all $x \in I$ but $f$ is not the identity, then there exists an interval $(\alpha, \beta)$ such that either $f(x)<x$ or $f(x)>x$ for all $x \in(\alpha, \beta)$ and $f((\alpha, \beta))=(\alpha, \beta)$. Since $f^{n}(x)=x$ for all $x \in(\alpha, \beta)$ and $f$ is comparable with identity, by Theorem $3.2 f(x)=x$ on $(\alpha, \beta)$, which is a contradiction. This forces that identity is the only order preserving homeomorphism of the identity function.

From Theorem 3.2, we can conclude that the non-commuting, non-comparable iterative roots of an order preserving homeomorphism are all different. We provide an illustrative example. The construction given in this example is based on Theorem 11.2.2 in [5].

Example 1. Consider the order preserving homeomorphism $F:[0,1] \rightarrow[0,1]$ defined by

$$
F(x)=\left\{\begin{array}{ccc}
4 x & \text { if } & x \in\left[0, \frac{1}{8}\right) \\
\frac{4}{3} x+\frac{1}{3} & \text { if } & x \in\left[\frac{1}{8}, \frac{1}{4}\right) \\
\frac{4}{9} x+\frac{5}{9} & \text { if } & x \in\left[\frac{1}{4}, 1\right] .
\end{array}\right.
$$

In order to construct iterative roots of this function, first we define a sequence of disjoint intervals whose union is $[0,1]$ and on each interval we define homeomorphism which serves as an iterative root of order 2 of $F$.

To start with, let $x_{0}=\frac{1}{8}$ and $x_{1}=\frac{1}{4}$. Define $x_{2 k}:=F\left(x_{2 k-2}\right), x_{2 k+1}:=F\left(x_{2 k-1}\right)$ for all $k \in \mathbb{N}$ and $x_{-(2 k+1)}:=F^{-1}\left(x_{-(2 k-1)}\right), x_{-2 k}:=F^{-1}\left(x_{-(2 k-2)}\right)$ for all $k \in \mathbb{N} \cup\{0\}$. Note that $x_{2}=F\left(x_{0}\right)=\frac{1}{2} ; x_{3}=F\left(x_{1}\right)=\frac{2}{3} ; x_{4}=F\left(x_{2}\right)=\frac{1}{2}\left(\frac{4}{9}\right)+\frac{5}{9} ; x_{5}=F\left(x_{3}\right)=\frac{2}{3}\left(\frac{4}{9}\right)+\frac{5}{9}$, in general 


$$
x_{2 k}=\frac{1}{2}\left(\frac{4}{9}\right)^{k-1}+\frac{5}{9} \sum_{i=0}^{k-2}\left(\frac{4}{9}\right)^{i}, x_{2 k+1}=\frac{2}{3}\left(\frac{4}{9}\right)^{k-1}+\frac{5}{9} \sum_{i=0}^{k-2}\left(\frac{4}{9}\right)^{i} \forall k \in \mathbb{N} \text {, }
$$

and $x_{-1}=F^{-1}\left(x_{1}\right)=\frac{1}{4}\left(\frac{1}{4}\right) ; x_{-2}=F^{-1}\left(x_{0}\right)=\frac{1}{8}\left(\frac{1}{4}\right) ; x_{-3}=F^{-1}\left(x_{-1}\right)=\frac{1}{4}\left(\frac{1}{4}\right)^{2} ; x_{-4}=F^{-1}\left(x_{-2}\right)=\frac{1}{8}\left(\frac{1}{4}\right)^{2}$, in iterative roots general

$$
x_{-(2 k+1)}=\frac{1}{4}\left(\frac{1}{4}\right)^{k+1}, x_{-2 k}=\frac{1}{8}\left(\frac{1}{4}\right)^{k} \forall k \in \mathbb{N} \cup\{0\} \text {. }
$$

Define $I_{k}=\left[x_{k}, x_{k+1}\right]$ for $k \in \mathbb{Z}$. Since $x_{2 k} \rightarrow 1, x_{2 k+1} \rightarrow 1, x_{-2 k} \rightarrow 0, x_{-(2 k+1)} \rightarrow 0$ as $k \rightarrow \infty$ we have $\cup_{k \in Z_{k}}=[0,1]$. Let $\phi_{0}: I_{0} \rightarrow I_{1}$ be the homeomorphism defined by $\square_{0}(x)=2 x$ for all $x \in I_{0}$. Now, define $\phi_{k}: I_{k} \rightarrow I_{k+1}$ by $\phi_{k}(x)=F \circ \phi_{k-1}^{-1}(x)$ for all $x \in I_{k}$ and $k \in \mathbb{N}$, also define $\phi_{-k}: I_{-k} \rightarrow I_{-(k-1)}$ by $\phi_{-k}(x)=\phi_{-(k-1)}^{-1} \circ F(x)$ for all $x \in I_{k}$ and $k \in \mathbb{N}$. Consider the homeomorphism $f:[0,1] \rightarrow[0,1]$ defined by $f(x)=\phi_{k}(x)$ if $x \in I_{k}$ for all $k \in \mathbb{Z}$. By calculation we can show that

$$
f(x)=\left\{\begin{array}{ccc}
2 x & \text { if } & x \in\left[0, \frac{1}{4}\right) \\
\frac{2}{3} x+\frac{1}{3} & \text { if } & x \in\left[\frac{1}{4}, 1\right] .
\end{array}\right.
$$

and $f^{2}(x)=F(x) \forall x \in[0,1]$. Now we construct another order preserving homeomorphism $g$ which do not subcommute and not comparable with $f$ but $g^{2}=F$. For this, let $\psi_{0}: I_{0} \rightarrow I_{1}$ be the homeomorphism defined by

$$
\psi_{0}(x)=\left\{\begin{array}{ccc}
x+\frac{1}{8} & \text { if } & x \in\left[\frac{1}{8}, \frac{3}{16}\right) \\
3 x-\frac{1}{4} & \text { if } & x \in\left[\frac{3}{16}, \frac{1}{4}\right] .
\end{array}\right.
$$

Now, define $\psi_{k}: I_{k} \rightarrow I_{k+1}$ by $\psi_{k}(x)=F \circ \psi_{k-1}^{-1}(x)$ for all $x \in I_{k}$ and $k \in \mathbb{N}$, also define $\psi_{-k}: I_{-k} \rightarrow I_{-(k-1)}$ by $\psi_{-k}(x)=\psi_{-(k-1)}^{-1} \circ F(x)$ for all $x \in I_{k}$ and $k \in \mathbb{N}$. Then the homeomorphism $g:[0,1] \rightarrow[0,1]$ defined by $g(x)=\psi_{k}(x)$ if $x \in I_{k}$ for all $k \in \mathbb{Z}$ satisfies $g^{2}(x)=F(x)$ for all $x \in[0,1]$. Since,

$$
\psi_{1}(x)=F \circ \psi_{0}^{-1}(x)=\left\{\begin{array}{lll}
\frac{4}{3} x+\frac{1}{6} & \text { if } & x \in\left[\frac{1}{4}, \frac{5}{16}\right) \\
\frac{4}{9} x+\frac{4}{9} & \text { if } & x \in\left[\frac{5}{16}, \frac{1}{2}\right],
\end{array}\right.
$$

and

$$
\psi_{2}(x)=F \circ \psi_{1}^{-1}(x)=\left\{\begin{array}{ccc}
\frac{1}{3} x+\frac{1}{2} & \text { if } & x \in\left[\frac{1}{2}, \frac{7}{12}\right) \\
x+\frac{1}{9} & \text { if } & x \in\left[\frac{7}{12}, \frac{2}{3}\right],
\end{array}\right.
$$


AJMS

$26,1 / 2$

\section{0}

we observe that

$$
f\left(g\left(\frac{3}{16}\right)\right)=f\left(\psi_{0}\left(\frac{3}{16}\right)\right)=f\left(\frac{5}{16}\right)=\frac{13}{24}<g\left(f\left(\frac{3}{16}\right)\right)=\psi_{1}\left(\frac{3}{8}\right)=\frac{11}{18},
$$

and

$$
g\left(f\left(\frac{13}{32}\right)\right)=\psi_{2}\left(\frac{29}{48}\right)=\frac{103}{144}<f\left(g\left(\frac{13}{32}\right)\right)=f\left(\psi_{1}\left(\frac{13}{32}\right)\right)=f\left(\frac{45}{72}\right)=\frac{27}{36} .
$$

Moreover, $g\left(\frac{3}{16}\right)=\frac{5}{16}<f\left(\frac{3}{16}\right)=\frac{3}{8}$ and $f\left(\frac{5}{16}\right)=\frac{13}{24}<g\left(\frac{5}{16}\right)=\frac{7}{12}$. Thus we have two order preserving homeomorphisms $f$ and $g$ such that they are neither comparable nor subcommuting but $f^{2}=g^{2}=F$ and $f \neq g$.

\section{References}

[1] C. Babbage, Essay towards the calculus of functions I, Philos. Trans. R. Soc. Lond. 105 (1815) 389-423.

[2] U.T. Bödewadt, Zur iteration reeller funktionen, Math. Z. 49 (1944) 497-516.

[3] D. Głazowska, J. Matkowski, Subcommuting and commuting real homographic functions, J. Difference Equ. Appl. 22 (2016) 177-187.

[4] M. Kuczma, On the functional equation $\phi^{n}(x)=g(x)$, Ann. Polon. Math. 11 (1961) 161-175.

[5] M. Kuczma, B. Choczewski, R. Ger, Iterative functional equations, in: Encyclopedia of Mathematics and its Applications, Vol. 32, Cambridge University Press, Cambridge, 1990.

[6] L. Liu, W. Jarczyk, L. Li, W. Zhang, Iterative roots of piecewise monotonic functions of nonmonotonicity height not less than 2, Nonlinear Anal. 75 (1) (2012) 286-303.

[7] S. Łojasiewicz, Solution générale de l'équation fonctionelle $f(f(\cdots f(x) \cdots))=g(x)$, Ann. Soc. Polon. Math. 24 (1951) 88-91.

[8] N. Mcshane, On the periodicity of homeomorphisms of the real line, Amer. Math. Monthly 6 (1961) 562-563.

[9] A. Smajdor, On some special iteration groups, Fund. Math. 82 (1973) 67-74.

[10] M.C. Zdun, Note on commutable functions, Aequationes Math. 36 (1988) 153-164.

[11] M.C. Zdun, P. Solarz, Recent results on iteration theory: Iteration groups and semigroups in the real case, Aequationes Math. 87 (2014) 201-245.

[12] W. Zhang, P.M. functions, PM functions their characteristic intervals and iterative roots, Ann. Polon. Math. 65 (1997) 119-128.

\section{Corresponding author}

Murugan Suresh Kumar can be contacted at: sureshkumarmsu2009@gmail.com

For instructions on how to order reprints of this article, please visit our website:

www.emeraldgrouppublishing.com/licensing/reprints.htm

Or contact us for further details: permissions@emeraldinsight.com 